\title{
TABLE DES MATIÈRES
}

Avant-propos ... . . . . . . . . . . . . . . 5

I. LES PROBLÈMES. . . . . . . . . . . . . . . . . . . . . . 7

0. Introduction . . . . . . . . . . . . . . . . 7

1. Les premiers chercheurs . . . . . . . . . . . . . . . . 9

2. Siciliano . . . . . . . . . . . . . . . . . 13

3. Les «structuralistes» . . . . . . . . . . . . . . . . . . . 19

4. Le bilan . . . . . . . . . . . . . . . . . . . . . . . 28

5. Composition et structure . . . . . . . . . . . . . . . . 29

6. Les problèmes accessoires . . . . . . . . . . . . . . . . . 34

7. Les critères . . . . . . . . . . . . . . . . . . 38

II. LeS PRÉSUPPOSÉS . . . . . . . . . . . . . . . . . . . . 42

1. L'analyse structurale, sa démarche . . . . . . . . . . . . . 42

2. L'analyse structurale, son résultat . . . . . . . . . . . . . 45

3. L'analyse structurale, sa présentation . . . . . . . . . . . . 47

4. Restrictions. . . . . . . . . . . . . . . . . . 49

5. Le testament fictionnel . . . . . . . . . . . . . 51

6. Le testament au XVe siècle . . . . . . . . . . . . . 54

7. Définition du testament . . . . . . . . . . . . . . . . 57

8. Liste des abréviations et sigles employés . . . . . . . . . . . 62

III. ModElle DU TESTAMENT RÉEL . . . . . . . . . . . . . 63

1. Les constituants performatifs . . . . . . . . . . . 63

1.0 Introduction ............... . 63 
1.1 Les constituants contractuels . . . . . . . . . . . . . . 65

1.2 Les constituants opératifs . . . . . . . . . . . . . . 66

1.3 Les constituants identificatifs. . . . . . . . . . . . . . 68

2. Les constituants additifs . . . . . . . . . . . 71

2.0 Introduction . . . . . . . . . . . . . 71

2.1 Les constituants semi-opératifs . . . . . . . . . . . . . 78

2.2 Les constituants homologatifs . . . . . . . . . . . . . 84

2.3 Les constituants justificatifs (JustContr) . . . . . . . . . 87

2.4 Les constituants ornementaux . . . . . . . . . . . . . 93

2.5 Les constituants justificatifs (JustOp) . . . . . . . . . . 95

2.6 Les constituants restrictifs . . . . . . . . . . . . . . . 96

2.7 Les constituants qualificatifs . . . . . . . . . . . . . . 98

3. Les constituants supplémentaires . . . . . . . . . . . . . 101

4. Distribution des constituants . . . . . . . . . . . . . 104

4.0 Introduction . . . . . . . . . . . . . . . . 104

4.1 Ordre dans lequel se présentent les constituants . . . . . . 111

4.2 Présence et absence de constituants . . . . . . . . . . . 114

4.3 Fréquence des constituants . . . . . . . . . . . 114

4.4 Proportion des constituants . . . . . . . . . . . 120

5. Fonction des constituants . . . . . . . . . . . . . . . 121

IV. Structure du lais . . . . . . . . . . . . . . . . . . . . 124

0. Introduction . . . . . . . . . . . . . . . . 124

1. Les constituants performatifs . . . . . . . . . . . . 124

1.1 Les constituants contractuels et réflexifs . . . . . . . . . 124

1.2 Les constituants opératifs . . . . . . . . . . . . . . . 127

1.3 Les constituants identificatifs. . . . . . . . . . . . . . 131

2. Les constituants additifs . . . . . . . . . . . . . . . 132

2.1 Les constituants semi-opératifs . . . . . . . . . . 132

2.2 Les constituants homologatifs . . . . . . . . . . . 133

2.3 Les constituants justificatifs (JustContr) . . . . . . . . . 134

2.4 Les constituants ornementaux . . . . . . . . . . . . 135

2.5 Les constituants justificatifs (JustOp) . . . . . . . . . 136

2.6 Les constituants restrictifs . . . . . . . . . . . . . . . 139

2.7 Les constituants qualificatifs . . . . . . . . . . . . . . 141

3. Les constituants supplémentaires . . . . . . . . . . . . . 148

4. Distribution des constituants . . . . . . . . . . . 152

4.0 Introduction . . . . . . . . . . . . 152 
4.1 Ordre dans lequel se présentent les constituants . . . . . . 153

4.2 Présence et absence de constituants . . . . . . . . . . . 154

4.3 Fréquence des constituants . . . . . . . . . . . 155

4.4 Proportion des constituants . . . . . . . . . . 157

V. Structure du Testament $\ldots \ldots \ldots$

1. Les constituants performatifs . . . . . . . . . . . . 160

1.1 Les constituants contractuels et réflexifs . . . . . . . . . 160

1.2 Les constituants opératifs . . . . . . . . . . . . . 166

1.3 Les constituants identificatifs. . . . . . . . . . . . . 177

2. Les constituants additifs . . . . . . . . . . . . 178

2.1 Les constituants semi-opératifs . . . . . . . . . . . . . 178

2.2 Les constituants homologatifs . . . . . . . . . . . . . 182

2.3 Les constituants justificatifs (JustContr) . . . . . . . . 184

2.4 Les constituants ornementaux . . . . . . . . . . . . . 186

2.5 Les constituants justificatifs (JustOp) . . . . . . . . 187

2.6 Les constituants restrictifs . . . . . . . . . . . . . . 195

2.7 Les constituants qualificatifs . . . . . . . . . . . . 200

3. Les constituants supplémentaires . . . . . . . . . . . 211

4. Distribution des constituants . . . . . . . . . . . . . . 224

4.0 Introduction . . . . . . . . . . . . . . . . 224

4.1 Ordre dans lequel se présentent les constituants . . . . . . 225

4.2 Présence et absence de constituants . . . . . . . . . . . 228

4.3 Fréquence des constituants . . . . . . . . . . . 229

4.4 Proportion des constituants . . . . . . . . . . . . . 232

VI. Caractìre fictionnel du lais et du testament . . . . . . 236

0. Introduction . . . . . . . . . . . . . 236

1. Fictivité et fictionnalité . . . . . . . . . . . . . . . . 239

2. Le signal fictionnel est un élément infractionnel . . . . . . . 241

3.1 Signaux fictionnels extratextuels . . . . . . . . . . . . 246

3.2 Signaux littéraires intrinsèques . . . . . . . . . . . . . . 249

4. Signaux fictionnels référentiels . . . . . . . . . . . . . 253

4.0 Introduction . . . . . . . . . . . . 253

4.1 Les legs factices . . . . . . . . . . . . . . . 255

4.2 Les faux légataires . . . . . . . . . . . . . . 261 
4.3 Absurdités . . . . . . . . . . . . . . . . . . . . 264

4.4 Absence de signaux fictionnels référentiels dans les parties non-opératives . . . . . . . . . . . . . 267

5. Les faits structuraux infractionnels . . . . . . . . . . . . 270

5.1 L'ordre dans lequel les constituants se présentent . . . . . 270

5.2 Présence et absence de constituants . . . . . . . . . . . 271

5.3 Fréquence des constituants . . . . . . . . . . . . 272

5.4 Proportion des constituants . . . . . . . . . . . . . 273

VII. Conclusions et PERSPECTIVES . . . . . . . . . . . . . . 274

0. Introduction . . . . . . . . . . . . . . . . 274

1. La discussion de la composition/structure . . . . . . . . . . 274

1.1 Macrostructure . . . . . . . . . . . . . . 274

1.2 La texture du Testament . . . . . . . . . . . . . . . 277

2. Interprétation des faits structuraux . . . . . . . . 281

2.1 A la recherche du sens général . . . . . . . . . 281

2.2 Nature humaine et condition humaine . . . . . . . . . 285

2.3 Le fonctionnement littéraire . . . . . . . . . . . . 293

3. Perspectives théoriques . . . . . . . . . . . . . . . 297

3.1 La fictionnalité . . . . . . . . . . . . . . . . 297

3.2 Cohérence . . . . . . . . . . . . . . . 302

3.3 Modèle descriptif . . . . . . . . . . . . 305

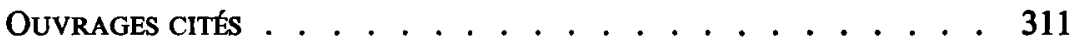

INDEX DES DÉFINITIONS $\ldots \ldots \ldots$ 